\title{
On Controlled Rectangular Metric Spaces and an Application
}

\author{
Nayab Alamgir, ${ }^{1}$ Quanita Kiran, ${ }^{2}$ Hassen Aydi $\mathbb{D}^{3},{ }^{3,4,5}$ and Yaé Ulrich Gaba ${ }^{6}{ }^{6,7,8}$ \\ ${ }^{1}$ School of Natural Sciences, National University of Sciences and Technology (NUST), Sector H-12, Islamabad, Pakistan \\ ${ }^{2}$ School of Electrical Engineering and Computer Science (SEECS), National University of Sciences and Technology (NUST), Sector H- \\ 12, Islamabad, Pakistan \\ ${ }^{3}$ Institut Supérieur d'Informatique et des Techniques de Communication, Université de Sousse, H. Sousse 4000, Tunisia \\ ${ }^{4}$ Department of Mathematics and Applied Mathematics, Sefako Makgatho Health Sciences University, Ga-Rankuwa, South Africa \\ ${ }^{5}$ China Medical University Hospital, China Medical University, Taichung, 40402, Taiwan \\ ${ }^{6}$ Quantum Leap Africa (QLA), AIMS Rwanda Centre, Remera Sector KN 3, Kigali, Rwanda \\ ${ }^{7}$ Institut de Mathématiques et de Sciences Physiques (IMSP/UAC), Laboratoire de Topologie Fondamentale, Computationnelle et \\ leurs Applications (Lab-ToFoCApp), BP 613, Porto-Novo, Benin \\ ${ }^{8}$ African Center for Advanced Studies, P.O. Box 4477, Yaounde, Cameroon
}

Correspondence should be addressed to Hassen Aydi; hassen.aydi@isima.rnu.tn

Received 6 March 2021; Revised 9 April 2021; Accepted 12 April 2021; Published 30 April 2021

Academic Editor: Tuncer Acar

Copyright (C) 2021 Nayab Alamgir et al. This is an open access article distributed under the Creative Commons Attribution License, which permits unrestricted use, distribution, and reproduction in any medium, provided the original work is properly cited.

\begin{abstract}
In this paper, we introduce the notion of controlled rectangular metric spaces as a generalization of rectangular metric spaces and rectangular $b$-metric spaces. Further, we establish some related fixed point results. Our main results extend many existing ones in the literature. The obtained results are also illustrated with the help of an example. In the last section, we apply our results to a common real-life problem in a general form by getting a solution for the Fredholm integral equation in the setting of controlled rectangular metric spaces.
\end{abstract}

\section{Introduction}

The fixed point theory is a growing and exciting field of mathematics with a variety of variant applications in mathematical sciences, proposing newer applications in discrete dynamics and super fractals. The fixed point theory is a fundamental tool to various theoretical and applied fields, such as variational and linear inequalities, the approximation theory, nonlinear analysis, integral and differential equations and inclusions, the dynamic systems theory, mathematics of fractals, mathematical economics (game theory, equilibrium problems, and optimization problems), and mathematical modeling; see [1-3]. In particular, fixed point techniques have been applied in such diverse fields; see $[4,5]$. There are particular real-life problems, whose statements are fairly easy to understand, which can be argued using some versions of fixed point theorems; see $[6,7]$.
The notion of extended $b$-metric spaces was introduced by Kamran et al. [9] as a generalization of metric spaces and $b$-metric spaces $[10,11]$. This metric type space has been generalized in several directions (for instance, controlled metric spaces [12], double controlled metric spaces [13], and others [14-19]). In a different perception, Branciari [20] proposed rectangular metric spaces. In the same order, Asim et al. [21] included a control function to initiate the concept of extended rectangular $b$-metric spaces as a generalization of rectangular $b$-metric spaces [22]. In [23], Mlaiki et al. introduced controlled rectangular $b$-metric spaces, which generalize rectangular metric spaces and rectangular $b$-metric spaces.

In this paper, our goal is to introduce the notion of controlled rectangular metric spaces, which is different from controlled rectangular $b$-metric spaces, and generalize rectangular metric spaces as well as rectangular $b$ -metric spaces. Further, we prove some fixed point 
results on such spaces as a generalization of many preexisting results in the literature. Also, we give examples for the justification of our results. In the last, as an application, we give an existence theorem for the Fredholm integral equation in the setting of controlled rectangular metric spaces.

\section{Preliminaries}

In this section, we collect some basic concepts related to our main results.

Definition 1 [22]. A mapping $d_{\zeta}: \mathscr{M} \times \mathscr{M} \longrightarrow[0, \infty)$ on a nonempty set $\mathscr{M}$ is called a rectangular $b$-metric space, if there exists a constant $s \geq 1$ such that for all $m_{1}, m_{2} \in \mathscr{M}$ and all distinct $\mu_{1}, \mu_{2} \in \mathscr{M}$ different from $m_{1}$ and $m_{2}$, the following axioms are satisfied:

(i) $d_{\zeta}\left(m_{1}, m_{2}\right)=0$ iff $m_{1}=m_{2}$

(ii) $d_{\zeta}\left(m_{1}, m_{2}\right)=d_{\zeta}\left(m_{2}, m_{1}\right)$

(iii) $d_{\zeta}\left(m_{1}, m_{2}\right) \leq s\left[d_{\zeta}\left(m_{1}, \mu_{1}\right)+d_{\zeta}\left(\mu_{1}, \mu_{2}\right)+d_{\zeta}\left(\mu_{2}\right.\right.$ $\left.m_{2}\right)$ ]

In this case, the pair $\left(\mathscr{M}, d_{\zeta}\right)$ is called a rectangular $b$ -metric space.

Definition 2 [9]. Let $\mathscr{M}$ be a nonempty set and $\zeta: \mathscr{M} \times \mathscr{M}$ $\rightarrow[1, \infty)$ be a mapping. Then, a mapping $d_{\zeta}: \mathscr{M} \times \mathscr{M} \longrightarrow$ $[0, \infty)$ is called an extended $b$-metric, if for all $m_{1}, m_{2}, m_{3}$ $\in \mathscr{M}$, it satisfies the following axioms:

(i) $d_{\zeta}\left(m_{1}, m_{2}\right)=0$ iff $m_{1}=m_{2}$

(ii) $d_{\zeta}\left(m_{1}, m_{2}\right)=d_{\zeta}\left(m_{2}, m_{1}\right)$

(iii) $d_{\zeta}\left(m_{1}, m_{3}\right) \leq \zeta\left(m_{1}, m_{3}\right)\left[d_{\zeta}\left(m_{1}, m_{2}\right)+d_{\zeta}\left(m_{2}\right.\right.$ $\left.\left.m_{3}\right)\right]$

The pair $\left(\mathscr{M}, d_{\zeta}\right)$ is called an extended $b$-metric space.

Definition 3 [21]. A mapping $d_{\zeta}: \mathscr{M} \times \mathscr{M} \longrightarrow[0, \infty)$ on a nonempty set $\mathscr{M}$ is called an extended rectangular $b$ -metric space, if for all $m_{1}, m_{2} \in \mathscr{M}$ and all distinct $\mu_{1}$, $\mu_{2} \in \mathscr{M}$ different from $m_{1}$ and $m_{2}$, the following axioms are satisfied:

(i) $d_{\zeta}\left(m_{1}, m_{2}\right)=0$ iff $m_{1}=m_{2}$

(ii) $d_{\zeta}\left(m_{1}, m_{2}\right)=d_{\zeta}\left(m_{2}, m_{1}\right)$

(iii) $d_{\zeta}\left(m_{1}, m_{2}\right) \leq \zeta\left(m_{1}, m_{2}\right)\left[d_{\zeta}\left(m_{1}, \mu_{1}\right)+d_{\zeta}\left(\mu_{1}, \mu_{2}\right)\right.$ $\left.+d_{\zeta}\left(\mu_{2}, m_{2}\right)\right]$

where $\zeta: \mathscr{M} \times \mathscr{M} \longrightarrow[1, \infty)$ is a mapping. In this case, the pair $\left(\mathscr{M}, d_{\zeta}\right)$ is called an extended rectangular $b$-metric space.

Note that the topology of rectangular metric spaces need not be Hausdorff. For more examples, see the papers of Sarma et al. [24] and Samet [25]. The topological structure of rectangular metric spaces is not compatible with the topology of classic metric spaces; see Example 7 in the paper of Suzuki [26]. In the same direction, extended rectangular $b$-metric spaces cannot be Hausdorff.

Definition 4 [23]. A mapping $d_{\zeta}: \mathscr{M} \times \mathscr{M} \longrightarrow[0, \infty)$ on a nonempty set $\mathscr{M}$ is called a controlled rectangular $b$-metric space, if for all distinct $m_{1}, m_{2}, \mu_{1}, \mu_{2} \in \mathscr{M}$, the following axioms are satisfied:

(i) $d_{\zeta}\left(m_{1}, m_{2}\right)=0$ iff $m_{1}=m_{2}$

(ii) $d_{\zeta}\left(m_{1}, m_{2}\right)=d_{\zeta}\left(m_{2}, m_{1}\right)$

(iii) $d_{\zeta}\left(m_{1}, m_{2}\right) \leq \zeta\left(m_{1}, m_{2}, \mu_{1}, \mu_{2}\right)\left[d_{\zeta}\left(m_{1}, \mu_{1}\right)+d_{\zeta}(\right.$ $\left.\left.\mu_{1}, \mu_{2}\right)+d_{\zeta}\left(\mu_{2}, m_{2}\right)\right]$

where $\zeta: \mathscr{M}^{4} \longrightarrow[1, \infty)$ is a mapping. In this case, the pair $\left(\mathscr{M}, d_{\zeta}\right)$ is called a controlled rectangular $b$-metric space.

As a generalization of metric spaces, Mlaiki et al. in [12] introduced the concept of controlled metric spaces as follows.

Definition 5 [12]. Let $\mathscr{M}$ be a nonempty set and $\zeta: \mathscr{M} \times \mathscr{M}$ $\longrightarrow[1, \infty)$. Then, a mapping $d_{\zeta}: \mathscr{M} \times \mathscr{M} \longrightarrow[0, \infty)$ is called a controlled metric, if for all $m_{1}, m_{2}, m_{3} \in \mathscr{M}$, it satisfies the following axioms:

(i) $d_{\zeta}\left(m_{1}, m_{2}\right)=0$ iff $m_{1}=m_{2}$

(ii) $d_{\zeta}\left(m_{1}, m_{2}\right)=d_{\zeta}\left(m_{2}, m_{1}\right)$

(iii) $d_{\zeta}\left(m_{1}, m_{3}\right) \leq \zeta\left(m_{1}, m_{2}\right) d_{\zeta}\left(m_{1}, m_{2}\right)+\zeta\left(m_{2}, m_{3}\right.$ )$d_{\zeta}\left(m_{2}, m_{3}\right)$

The pair $\left(\mathscr{M}, d_{\zeta}\right)$ is called a controlled metric space.

Note that Definition 5 generalizes $b$-metric spaces and is different from Definition 2.

Example 1 [12]. Let $\mathscr{M}=\{1,2, \cdots\}$. Define $d_{\zeta}: \mathscr{M} \times \mathscr{M} \longrightarrow$ $[0, \infty)$ as

$d_{\zeta}(x, y)= \begin{cases}0, & \text { if } m_{1}=m_{2}, \\ \frac{1}{m_{1}}, & \text { if } m_{1} \text { is even and } m_{2} \text { is odd, } \\ \frac{1}{m_{2}}, & \text { if } m_{1} \text { is odd and } m_{2} \text { is even, } \\ 1, & \text { otherwise. }\end{cases}$

Hence, $\left(\mathscr{M}, d_{\zeta}\right)$ is a controlled metric space, where $\zeta$ 
$: \mathscr{M} \times \mathscr{M} \longrightarrow[1, \infty)$ is defined as

$$
\zeta\left(m_{1}, m_{2}\right)= \begin{cases}m_{1}, & \text { if } m_{1} \text { is even and } m_{2} \text { is odd } \\ m_{2}, & \text { if } m_{1} \text { is odd and } m_{2} \text { is even } \\ 1, & \text { otherwise }\end{cases}
$$

\section{Main Results}

In this section, we introduce the notion of controlled rectangular metric spaces. Also, we establish some fixed point results.

Definition 6. A mapping $d_{\zeta}: \mathscr{M} \times \mathscr{M} \longrightarrow[0, \infty)$ on a nonempty set $\mathscr{M}$ is called a controlled rectangular metric space, if for all $m_{1}, m_{2} \in \mathscr{M}$ and all distinct $\mu_{1}, \mu_{2} \in \mathscr{M}$ different from $m_{1}$ and $m_{2}$, the following axioms are satisfied:

(i) $d_{\zeta}\left(m_{1}, m_{2}\right)=0$ iff $m_{1}=m_{2}$

(ii) $d_{\zeta}\left(m_{1}, m_{2}\right)=d_{\zeta}\left(m_{2}, m_{1}\right)$

(iii) $d_{\zeta}\left(m_{1}, m_{2}\right) \leq \zeta\left(m_{1}, \mu_{1}\right) d_{\zeta}\left(m_{1}, \mu_{1}\right)+\zeta\left(\mu_{1}, \mu_{2}\right) d_{\zeta}($ $\left.\mu_{1}, \mu_{2}\right)+\zeta\left(\mu_{2}, m_{2}\right) d_{\zeta}\left(\mu_{2}, m_{2}\right)$

where $\zeta: \mathscr{M} \times \mathscr{M} \longrightarrow[1, \infty)$ is a mapping, In this case, the pair $\left(\mathscr{M}, d_{\zeta}\right)$ is called a controlled rectangular metric space.

\section{Remark 7.}

(i) Every rectangular metric space and rectangular $b$ -metric is a controlled rectangular metric space

(ii) Clearly, Definition 6 is different from Definition 4

(iii) Every controlled metric space is a controlled rectangular metric space, but its converse is not true in general. See the following example

Example 2. Let $\mathscr{M}=[0, \infty)$. Define a mapping $d_{\zeta}: \mathscr{M} \times \mathscr{M}$ $\longrightarrow[0, \infty)$ by

$$
d_{\zeta}\left(m_{1}, m_{2}\right)= \begin{cases}0, & \text { if } m_{1}=m_{2}, \\ \frac{1}{m_{1}}, & \text { if } m_{1} \geq 1 \text { and } m_{2} \in[0,1), \\ \frac{1}{m_{2}}, & \text { if } m_{2} \geq 1 \text { and } m_{1} \in[0,1), \\ 1, & \text { otherwise. }\end{cases}
$$

Then, $d_{\zeta}$ is a controlled rectangular metric space, where $\zeta: \mathscr{M} \times \mathscr{M} \longrightarrow[1, \infty)$ is a mapping defined as

$$
\zeta\left(m_{1}, m_{2}\right)= \begin{cases}m_{1}, & \text { if } m_{1}, m_{2} \geq 1 \\ 1, & \text { otherwise }\end{cases}
$$

Clearly, $\left(\mathscr{M}, d_{\zeta}\right)$ is not a controlled metric space if we take $m_{1}, m_{3} \geq 1$ and $m_{2} \in[0,1)$. Then, $d_{\zeta}\left(m_{1}, m_{3}\right)=1, d_{\zeta}$ $\left(m_{1}, m_{2}\right)=1 / m_{1}, \quad d_{\zeta}\left(m_{2}, m_{3}\right)=1 / m_{3}, \quad \zeta\left(m_{1}, m_{2}\right)=1$, and $\zeta\left(m_{2}, m_{3}\right)=1$. Here, the triangle inequality is not satisfied:

$$
\begin{aligned}
d_{\zeta}\left(m_{1}, m_{3}\right)=1 & >\zeta\left(m_{1}, m_{2}\right) d_{\zeta}\left(m_{1}, m_{2}\right) \\
& +\zeta\left(m_{2}, m_{3}\right) d_{\zeta}\left(m_{2}, m_{3}\right)=\frac{1}{m_{1}}+\frac{1}{m_{3}} .
\end{aligned}
$$

Example 3. Let $\mathscr{M}=\{1,2,3,4\}$. Define $d_{\zeta}: \mathscr{M} \times \mathscr{M} \longrightarrow[o$, $\infty)$ as

$$
\begin{aligned}
d_{\zeta}(1,1) & =d_{\zeta}(2,2)=d_{\zeta}(3,3)=d_{\zeta}(4,4)=0, \\
d_{\zeta}(1,2) & =d_{\zeta}(2,1)=d_{\zeta}(2,3)=d_{\zeta}(3,2)=d_{\zeta}(3,4)=d_{\zeta}(4,3) \\
& =d_{\zeta}(1,3)=d_{\zeta}(3,1)=80, \\
d_{\zeta}(1,4) & =d_{\zeta}(4,1)=1000, \\
d_{\zeta}(2,4) & =d_{\zeta}(4,2)=450 .
\end{aligned}
$$

Then, $\left(\mathscr{M}, d_{\zeta}\right)$ is a controlled rectangular metric space with $\zeta: \mathscr{M} \times \mathscr{M} \longrightarrow[1, \infty)$ defined as $\zeta\left(m_{1}, m_{2}\right)=\max \{$ $\left.m_{1}, m_{2}\right\}+2$, for all $m_{1}, m_{2} \in \mathscr{M}$. However, $\left(\mathscr{M}, d_{\zeta}\right)$ is not a rectangular metric space; for instance, notice

$$
d_{\zeta}(1,4)=1000>d_{\zeta}(1,2)+d_{\zeta}(2,3)+d_{\zeta}(3,4)=240 .
$$

The concepts of convergence, Cauchyness, and completeness can simply be generalized in terms of controlled rectangular metric spaces.

Definition 8 . Let $\left(\mathscr{M}, d_{\zeta}\right)$ be a controlled rectangular metric space. Then,

(i) A sequence $\left\{m_{n}\right\}$ in $\left(\mathscr{M}, d_{\zeta}\right)$ is said to be convergent to $m \in \mathscr{M}$, if $\lim _{n \longrightarrow \infty} d_{\zeta}\left(m_{n}, m\right)=0$

(ii) A sequence $\left\{m_{n}\right\}$ in $\left(\mathscr{M}, d_{\zeta}\right)$ is called a Cauchy sequence, if $\lim _{n, r \longrightarrow \infty} d_{\zeta}\left(m_{n}, m_{r}\right)=0$

(iii) $\left(\mathscr{M}, d_{\zeta}\right)$ is called a complete controlled rectangular metric space, if every Cauchy sequence in $\mathscr{M}$ is convergent to some point of $\mathscr{M}$

Definition 9. Let $\left(\mathscr{M}, d_{\zeta}\right)$ be a controlled rectangular metric space. Let $m \in \mathscr{M}$ and $\tau>0$. Then,

(i) The open ball $B(m, \tau)$ is define as

$$
B(m, \tau)=\left\{m_{1} \in \mathscr{M}, d_{\zeta}\left(m, m_{1}\right)<\tau\right\} .
$$

(ii) The mapping $\mathbf{f}: \mathscr{M} \longrightarrow \mathscr{M}$ is called continuous at $m \in \mathscr{M}$, if for $v>0$, there is $v>0$ such that $\mathbf{f}(B(m$, 
$v)) \subseteq B(\mathbf{f}(m), v)$. Thus, if $\mathbf{f}$ is continuous at $m$, then for any sequence $\left\{m_{n}\right\}$ converging to $m$, we have $\lim _{n \rightarrow \infty} \mathbf{f} m_{n}=\mathbf{f} m$

Note that a rectangular $b$-metric space is not continuous in general, and it is the same for controlled rectangular metric spaces.

Lemma 10. Let $\left(\mathscr{M}, d_{\zeta}\right)$ be a controlled rectangular metric space and $\left\{m_{n}\right\}$ be a Cauchy sequence in $\mathscr{M}$ such that $m_{n}$ $\neq m_{r}$, whenever $n \neq r$. If $\lim _{n, r \rightarrow \infty} \zeta\left(m_{n}, m_{r}\right)<\infty$ for all $m_{n}, m_{r} \in \mathscr{M}$, then $\left\{m_{n}\right\}$ has a unique limit.

Proof. Suppose that a sequence $\left\{m_{n}\right\}$ in $\mathscr{M}$ has two limit points $\mu, v \in \mathscr{M}$, that is, $\lim _{n \rightarrow \infty} m_{n}=\mu$ and $\lim _{n \rightarrow \infty} m_{n}$ $=v \cdot\left\{m_{n}\right\}$ is a Cauchy sequence for $m_{n} \neq m_{r}$, whenever $n$ $\neq r$. Hence, from condition (iii) of Definition 6 , we have

$$
\begin{gathered}
d_{\zeta}(\mu, v) \leq \zeta\left(\mu, m_{n}\right) d_{\zeta}\left(\mu, m_{n}\right)+\zeta\left(m_{n}, m_{r}\right) d_{\zeta}\left(m_{n}, m_{r}\right) \\
+\zeta\left(m_{r}, v\right) d_{\zeta}\left(m_{r}, v\right) \longrightarrow 0 \text { as } n, r \longrightarrow \infty
\end{gathered}
$$

This implies that

$$
d_{\zeta}(\mu, v)=0
$$

Hence, $\left\{m_{n}\right\}$ has a unique limit point in $\mathscr{M}$.

Definition 11. Let $\left(\mathscr{M}, d_{\zeta}\right)$ be a controlled rectangular metric space. Then,

(i) For a mapping f : $\mathscr{M} \longrightarrow \mathscr{M}$, we define

$$
\begin{gathered}
\mathcal{O}(m, n)=\left\{m, \mathbf{f} m, \cdots, \mathbf{f}^{n} m\right\} \\
\mathcal{O}(m, \infty)=\left\{m, \mathbf{f} m, \cdots, \mathbf{f}^{n} m, \cdots\right\}
\end{gathered}
$$

where $m \in \mathscr{M}$ and $n \in \mathbb{N}$. The $\mathcal{O}(m, \infty)$ is called an orbit of $\mathbf{f}$

(ii) A mapping f : $\mathscr{M} \longrightarrow \mathscr{M}$ is called f-orbitally continuous, if $\lim _{k \longrightarrow \infty} \mathbf{f}_{k}^{n} m=m$ implies $\lim _{k \longrightarrow \infty} \mathbf{f}\left(\mathbf{f}_{k}^{n} m\right)$ $=\mathbf{f} m$, where $m \in \mathscr{M}$

(iii) A mapping f : $\mathscr{M} \longrightarrow \mathscr{M}$ is called f-orbitally complete, if every Cauchy sequence in $\mathcal{O}(m, \infty)$ is convergent in $\mathscr{M}$

Our main result is similar to the Banach contraction principle in the setting of controlled rectangular metric spaces. Throughout this section, for a mapping $\mathbf{f}: \mathscr{M} \longrightarrow$ $\mathscr{M}$ and $m \in \mathscr{M}$, we consider an orbit $\mathcal{O}(m, \infty)$.

Theorem 12. Let $\mathbf{f}: \mathscr{M} \longrightarrow \mathscr{M}$ be a mapping on a controlled rectangular metric space $\left(\mathscr{M}, d_{\zeta}\right)$. Suppose that the following axioms hold: (i) For all $m_{1}, m_{2} \in \mathscr{M}$, we have

$$
d_{\zeta}\left(\mathbf{f} m_{1}, \mathbf{f} m_{2}\right) \leq \lambda d_{\zeta}\left(m_{1}, m_{2}\right)
$$

where $\lambda \in[0,1)$.

(ii) $\sup _{q \geq 1} \lim _{i \rightarrow \infty} \zeta\left(m_{i}, m_{q}\right)\left(\zeta\left(m_{i+1}, m_{i+2}\right) / \zeta\left(m_{i-1}, m_{i}\right.\right.$ )) $\lambda<1$, for any $m_{n} \in \mathscr{M}$

(iii) $\left(\mathscr{M}, d_{\zeta}\right)$ is $\mathbf{f}$-orbitally complete

(iv) $\mathbf{f}$ is orbitally continuous

(v) For each $m \in \mathscr{M}, \lim _{n \rightarrow \infty} \varsigma\left(m_{n}, \mu\right)$ and $\lim _{n \rightarrow \infty} \varsigma$ $\left(\mu, m_{n}\right)$ exist and are finite

Then, $\mathbf{f}$ has a unique fixed point in $\mathscr{M}$.

Proof. Consider an arbitrary point $m_{0} \in \mathscr{M}$, and define an iterative sequence $\left\{m_{n}\right\}$ over $m_{0}$ as follows:

$$
m_{1}=\mathbf{f} m_{0}, m_{2}=\mathbf{f} m_{1}=\mathbf{f}\left(\mathbf{f} m_{0}\right)=\mathbf{f}^{2} m_{0}, \cdots, m_{n}=\mathbf{f}^{n} m_{0}, \cdots
$$

From equation (12), we have

$d_{\zeta}\left(m_{1}, m_{2}\right)=d_{\zeta}\left(\mathbf{f} m_{0}, \mathbf{f}^{2} m_{0}\right) \leq \lambda d_{\zeta}\left(m_{0}, \mathbf{f} m_{0}\right)=d_{\zeta}\left(m_{0}, m_{1}\right)$

Recursively, we have

$$
\begin{aligned}
d_{\zeta}\left(m_{n}, m_{n+1}\right)= & d_{\zeta}\left(\mathbf{f}^{n} m_{0}, \mathbf{f}^{n+1} m_{0}\right) \leq \lambda d_{\zeta}\left(\mathbf{f}^{n-1} m_{0}, \mathbf{f}^{n} m_{0}\right) \\
& \leq \lambda^{2} d_{\zeta}\left(\mathbf{f}^{n-2} m_{0}, \mathbf{f}^{n-1} m_{0}\right) \cdots \\
& \leq \lambda^{n} d_{\zeta}\left(m_{0}, m_{1}\right) .
\end{aligned}
$$

That is,

$$
d_{\zeta}\left(m_{n}, m_{n+1}\right) \leq \lambda^{n} d_{\zeta}\left(m_{0}, m_{1}\right)
$$

By taking limit $n \longrightarrow \infty$, we get

$$
\begin{aligned}
\lim _{n \longrightarrow \infty} d_{\zeta}\left(m_{n}, m_{n+1}\right) & =0 \\
\lim _{n \longrightarrow \infty} d_{\zeta}\left(m_{n+1}, m_{n+2}\right) & =0 .
\end{aligned}
$$


Next, we show that $\left\{m_{n}\right\}$ is a Cauchy sequence. For this, we will take the following two cases.

Case 1. Let $\rho$ be odd, that is, $\rho=2 r+1$, where $r \geq 1$. Then, from condition (iii) of Definition 6 and equation (16) for $n+\rho>n$, we have

$$
\begin{aligned}
d_{\zeta}\left(m_{n}, m_{n+2 r+1}\right) \leq \zeta\left(m_{n}, m_{n+1}\right) d_{\zeta}\left(m_{n}, m_{n+1}\right)+\zeta \\
\quad \cdot\left(m_{n+1}, m_{n+2}\right) d_{\zeta}\left(m_{n+1}, m_{n+2}\right)+\zeta\left(m_{n+2}, m_{n+2 r+1}\right) \\
\quad \times d_{\zeta}\left(m_{n+2}, m_{n+2 r+1}\right) \leq\left[\zeta\left(m_{n}, m_{n+1}\right) \lambda^{n}+\zeta\right. \\
\left.\quad \cdot\left(m_{n+1}, m_{n+2}\right) \lambda^{n+1}\right] d_{\zeta}\left(m_{0}, m_{1}\right)+\zeta\left(m_{n+2}, m_{n+2 r+1}\right) d_{\zeta} \\
\quad \cdot\left(m_{n+2}, m_{n+2 r+1}\right) \leq\left[\zeta\left(m_{n}, m_{n+1}\right) \lambda^{n}+\zeta\right. \\
\left.\quad \cdot\left(m_{n+1}, m_{n+2}\right) \lambda^{n+1}\right] d_{\zeta}\left(m_{0}, m_{1}\right)+\zeta\left(m_{n+2}, m_{n+2 r+1}\right) \zeta \\
\quad \cdot\left(m_{n+2}, m_{n+3}\right) \times d_{\zeta}\left(m_{n+2}, m_{n+3}\right)+\zeta\left(m_{n+2}, m_{n+2 r+1}\right) \zeta \\
\quad \cdot\left(m_{n+3}, m_{n+4}\right) d_{\zeta}\left(m_{n+3}, m_{n+4}\right)+\zeta\left(m_{n+2}, m_{n+2 r+1}\right) \times \zeta \\
\quad \cdot\left(m_{n+4}, m_{n+2 r+1}\right) d_{\zeta}\left(m_{n+4}, m_{n+2 r+1}\right) \leq d_{\zeta} \\
\quad \cdot\left(m_{0}, m_{1}\right)\left[\zeta\left(m_{n}, m_{n+1}\right) \lambda^{n}+\zeta\left(m_{n+2}, m_{n+3}\right) \lambda^{n+1}\right] \\
\quad+d_{\zeta}\left(m_{0}, m_{1}\right) \times\left[\zeta\left(m_{n+2}, m_{n+2 r+1}\right) \zeta\left(m_{n+2}, m_{n+3}\right) \lambda^{n+2}\right. \\
\left.\quad+\zeta\left(m_{n+2}, m_{n+2 r+1}\right) \zeta\left(m_{n+3}, m_{n+4}\right) \lambda^{n+3}\right]+\cdots+d_{\zeta} \\
\quad \cdot\left(m_{0}, m_{1}\right) \zeta\left(m_{n+2}, m_{n+2 r+1}\right) \cdots \zeta\left(m_{n+2 r-2}, m_{n+2 n-1}\right) \lambda^{n+2 r-2} \\
\quad+\zeta\left(m_{n+2}, m_{n+2 r+1}\right) \cdots \zeta\left(m_{n+2 r-1}, m_{n+2 r}\right) \lambda^{n+2 r-1} \\
\quad+\zeta\left(m_{n+2}, m_{n+2 r+1}\right) \cdots \zeta\left(m_{n+2 r}, m_{n+2 r+1}\right) \lambda^{n+2 r} d_{\zeta}\left(m_{0}, m_{1}\right) \\
\quad \leq d_{\zeta}\left(m_{0}, m_{1}\right)\left[\zeta\left(m_{n}, m_{n+1}\right) \lambda^{n}+\zeta\left(m_{n+2}, m_{n+3}\right) \lambda^{n+1}\right] \\
\quad+\sum_{i=1}^{r-1} \prod_{j=1}^{i} \zeta\left(m_{n+2 j}, m_{n+2 r+1}\right) \times \zeta\left(m_{n+2 i}, m_{n+2 i+1}\right) \lambda^{n+2 i} d_{\zeta} \\
\quad \cdot\left(m_{0}, m_{1}\right)+\sum_{i=1}^{r-1} \prod_{j=1}^{i} \zeta\left(m_{n+2 j+1}, m_{n+2 r+1}\right) \zeta\left(m_{n+2 i+1}, m_{n+2 i+2}\right) \\
\quad \times \lambda^{n+2 i+1} d_{\zeta}\left(m_{0}, m_{1}\right)+\prod_{i=1}^{r-1} \zeta\left(m_{n+2 j}, m_{n+2 r+1}\right) \lambda^{n+2 r} d_{\zeta}\left(m_{0}, m_{1}\right)
\end{aligned}
$$

As

$$
\begin{aligned}
& \sum_{i=1}^{r-1} \prod_{j=1}^{i} \zeta\left(m_{n+2 j}, m_{n+2 r+1}\right) \zeta\left(m_{n+2 i}, m_{n+2 i+1}\right) \lambda^{n+2 i} \\
& \quad \leq \sum_{i=1}^{r-1} \prod_{j=1}^{i} \zeta\left(m_{2 j}, m_{n+2 r+1}\right) \zeta\left(m_{2 i}, m_{n+2 i+1}\right) \lambda^{2 i} \\
& \sum_{i=1}^{r-1} \prod_{j=1}^{i} \zeta\left(m_{n+2 j+1}, m_{n+2 r+1}\right) \zeta\left(m_{n+2 i+1}, m_{n+2 i+2}\right) \lambda^{n+2 i+1} \\
& \quad \leq \sum_{i=1}^{r-1} \prod_{j=1}^{i} \zeta\left(m_{2 j+1}, m_{n+2 r+1}\right) \zeta\left(m_{2 i+1}, m_{n+2 i+2}\right) \lambda^{2 i+1},
\end{aligned}
$$

therefore, we obtain

$$
\begin{aligned}
& d_{\zeta}\left(m_{n}, m_{n+2 r+1}\right) \leq d_{\zeta}\left(m_{0}, m_{1}\right)\left[\zeta\left(m_{n}, m_{n+1}\right) \lambda^{n}\right. \\
& \left.\quad+\zeta\left(m_{n+2}, m_{n+3}\right) \lambda^{n+1}\right]+\sum_{i=1}^{r-1} \prod_{j=1}^{i} \zeta\left(m_{2 j}, m_{n+2 r+1}\right) \\
& \quad \times \zeta\left(m_{2 i}, m_{2 i+1}\right) \lambda^{2 i} d_{\zeta}\left(m_{0}, m_{1}\right) \\
& \quad+\sum_{i=1}^{r-1} \prod_{j=1}^{i} \zeta\left(m_{2 j+1}, m_{n+2 r+1}\right) \zeta\left(m_{2 i+1}, m_{2 i+2}\right) \\
& \quad \times \lambda^{2 i+1} d_{\zeta}\left(m_{0}, m_{1}\right)+\prod_{i=1}^{r-1} \zeta\left(m_{n+2 j}, m_{n+2 r+1}\right) \lambda^{n+2 r} d_{\zeta}\left(m_{0}, m_{1}\right) .
\end{aligned}
$$

Since $\sup _{q \geq 1} \lim _{i \rightarrow \infty} \zeta\left(m_{i}, m_{q}\right)\left(\zeta\left(m_{i+1}, m_{i+2}\right) / \zeta\left(m_{i-1}\right.\right.$, $\left.\left.m_{i}\right)\right) \lambda<1$, the series

$$
\begin{gathered}
\sum_{i=1}^{\infty} \prod_{j=1}^{i} \zeta\left(m_{2 j}, m_{n+2 r+1}\right) \zeta\left(m_{2 i}, m_{2 i+1}\right) \lambda^{2 i}, \\
\sum_{i=1}^{\infty} \prod_{j=1}^{i} \zeta\left(m_{2 j+1}, m_{n+2 r+1}\right) \zeta\left(m_{2 i+1}, m_{2 i+2}\right) \lambda^{2 i+1},
\end{gathered}
$$

converge by the ratio test. Let

$$
\begin{aligned}
\mathcal{S} & =\sum_{i=1}^{\infty} \prod_{j=1}^{i} \zeta\left(m_{2 j}, m_{n+2 r+1}\right) \zeta\left(m_{2 i}, m_{2 i+1}\right) \lambda^{2 i}, \\
\mathcal{S}_{n} & =\sum_{i=1}^{n} \prod_{j=1}^{i} \zeta\left(m_{2 j}, m_{n+2 r+1}\right) \zeta\left(m_{2 i}, m_{2 i+1}\right) \lambda^{2 i}, \\
\mathcal{S}^{\prime} & =\sum_{i=1}^{\infty} \prod_{j=1}^{i} \zeta\left(m_{2 j+1}, m_{n+2 r+1}\right) \zeta\left(m_{2 i+1}, m_{2 i+2}\right) \lambda^{2 i+1}, \\
\mathcal{S}_{n}^{\prime} & =\sum_{i=1}^{n} \prod_{j=1}^{i} \zeta\left(m_{2 j+1}, m_{n+2 r+1}\right) \zeta\left(m_{2 i+1}, m_{2 i+2}\right) \lambda^{2 i+1} .
\end{aligned}
$$

Then, equation (22) takes the following form:

$$
\begin{aligned}
& d_{\zeta}\left(m_{n}, m_{n+2 r+1}\right) \leq d_{\zeta}\left(m_{0}, m_{1}\right)\left[\zeta\left(m_{n}, m_{n+1}\right) \lambda^{n}\right. \\
& \left.\quad+\zeta\left(m_{n+2}, m_{n+3}\right) \lambda^{n+1}\right]+d_{\zeta}\left(m_{0}, m_{1}\right)\left[\mathcal{S}_{r-1}-\mathcal{S}_{n+1}\right] d_{\zeta} \\
& \quad \cdot\left(m_{0}, m_{1}\right)+d_{\zeta}\left(m_{0}, m_{1}\right)\left[\mathcal{S}_{r-1}^{\prime}-\mathcal{S}_{n+1}^{\prime}\right] d_{\zeta}\left(m_{0}, m_{1}\right) \\
& \quad+\prod_{i=1}^{r-1} \zeta\left(m_{n+2 j}, m_{n+2 r+1}\right) \lambda^{n+2 r} d_{\zeta}\left(m_{0}, m_{1}\right) .
\end{aligned}
$$

By taking limit $n \longrightarrow \infty$ in equation (26), we get

$$
\lim _{n \longrightarrow \infty} d_{\zeta}\left(m_{n}, m_{n+2 r+1}\right)=0 .
$$


Case 2. Let $\rho$ be even, that is, $\rho=2 r$, where $r \geq 1$. Then, from condition (iii) of Definition 6 and equation (16) for $n+\rho>n$, we have

$$
\begin{aligned}
& d_{\zeta}\left(m_{n}, m_{n+2 r}\right) \leq \zeta\left(m_{n}, m_{n+1}\right) d_{\zeta}\left(m_{n}, m_{n+1}\right) \\
& +\zeta\left(m_{n+1}, m_{n+2}\right) d_{\zeta} .\left(m_{n+1}, m_{n+2}\right)+\zeta\left(m_{n+2}, m_{n+2 r}\right) \\
& \times d_{\zeta}\left(m_{n+2}, m_{n+2 r}\right) \leq\left[\zeta\left(m_{n}, m_{n+1}\right) \lambda^{n}\right. \\
& \left.+\zeta\left(m_{n+1}, m_{n+2}\right) \lambda^{n+1}\right] d_{\zeta}\left(m_{0}, m_{1}\right) \\
& +\zeta\left(m_{n+2}, m_{n+2 r}\right) d_{\zeta}\left(m_{n+2}, m_{n+2 r}\right) \leq\left[\zeta\left(m_{n}, m_{n+1}\right) \lambda^{n}\right. \\
& \left.+\zeta\left(m_{n+1}, m_{n+2}\right) \lambda^{n+1}\right] d_{\zeta}\left(m_{0}, m_{1}\right) \\
& +\zeta\left(m_{n+2}, m_{n+2 r}\right) \zeta\left(m_{n+2}, m_{n+3}\right) \times d_{\zeta}\left(m_{n+2}, m_{n+3}\right) \\
& +\zeta\left(m_{n+2}, m_{n+2 r}\right) \zeta\left(m_{n+3}, m_{n+4}\right) d_{\zeta}\left(m_{n+3}, m_{n+4}\right) \\
& +\zeta\left(m_{n+2}, m_{n+2 r}\right) \times \zeta\left(m_{n+4}, m_{n+2 r}\right) d_{\zeta}\left(m_{n+4}, m_{n+2 r}\right) \\
& \leq d_{\zeta}\left(m_{0}, m_{1}\right)\left[\zeta\left(m_{n}, m_{n+1}\right) \lambda^{n}+\zeta\left(m_{n+2}, m_{n+3}\right) \lambda^{n+1}\right] \\
& +d_{\zeta}\left(m_{0}, m_{1}\right) \times\left[\zeta\left(m_{n+2}, m_{n+2 r}\right) \zeta\left(m_{n+2}, m_{n+3}\right) \lambda^{n+2}\right. \\
& \left.+\zeta\left(m_{n+2}, m_{n+2 r}\right) \zeta\left(m_{n+3}, m_{n+4}\right) \lambda^{n+3}\right] \\
& +\cdots+d_{\zeta}\left(m_{0}, m_{1}\right) \zeta\left(m_{n+2}, m_{n+2 r}\right) \cdots \zeta \\
& \cdot\left(m_{n+2 r-4}, m_{n+2 n-3}\right) \lambda^{n+2 r-4}+\zeta\left(m_{n+2}, m_{n+2 r}\right) \cdots \zeta \\
& \cdot\left(m_{n+2 r-3}, m_{n+2 r-2}\right) \lambda^{n+2 r-3}+\zeta\left(m_{n+2}, m_{n+2 r}\right) \cdots \zeta \\
& \cdot\left(m_{n+2 r-2}, m_{n+2 r}\right) \lambda^{n+2 r-2} d_{\zeta}\left(m_{0}, m_{2}\right) \leq d_{\zeta} \\
& \cdot\left(m_{0}, m_{1}\right)\left[\zeta\left(m_{n}, m_{n+1}\right) \lambda^{n}+\zeta\left(m_{n+2}, m_{n+3}\right) \lambda^{n+1}\right] \\
& +\sum_{i=1}^{r-2} \prod_{j=1}^{i} \zeta\left(m_{n+2 j}, m_{n+2 r}\right) \times \zeta\left(m_{n+2 i}, m_{n+2 i+1}\right) \lambda^{n+2 i} d_{\zeta} \\
& \cdot\left(m_{0}, m_{1}\right)+\sum_{i=1}^{r-2} \prod_{j=1}^{i} \zeta\left(m_{n+2 j+1}, m_{n+2 r}\right) \zeta \\
& \cdot\left(m_{n+2 i+1}, m_{n+2 i+2}\right) \times \lambda^{n+2 i+1} d_{\zeta}\left(m_{0}, m_{1}\right) \\
& +\prod_{i=1}^{r-1} \zeta\left(m_{n+2 j}, m_{n+2 r}\right) \lambda^{n+2 r-2} d_{\zeta}\left(m_{0}, m_{2}\right)
\end{aligned}
$$

As

$$
\begin{gathered}
\sum_{i=1}^{r-2} \prod_{j=1}^{i} \zeta\left(m_{n+2 j}, m_{n+2 r}\right) \zeta\left(m_{n+2 i}, m_{n+2 i+1}\right) \lambda^{n+2 i} \\
\quad \leq \sum_{i=1}^{r-2} \prod_{j=1}^{i} \zeta\left(m_{2 j}, m_{n+2 r}\right) \zeta\left(m_{2 i}, m_{2 i+1}\right) \lambda^{2 i}
\end{gathered}
$$

$$
\begin{aligned}
& \sum_{i=1}^{r-2} \prod_{j=1}^{i} \zeta\left(m_{n+2 j+1}, m_{n+2 r+1}\right) \zeta\left(m_{n+2 i+1}, m_{n+2 i+2}\right) \lambda^{n+2 i+1} \\
& \quad \leq \sum_{i=1}^{r-2} \prod_{j=1}^{i} \zeta\left(m_{2 j+1}, m_{n+2 r+1}\right) \zeta\left(m_{2 i+1}, m_{2 i+2}\right) \lambda^{2 i+1}
\end{aligned}
$$

therefore, we obtain

$$
\begin{aligned}
& d_{\zeta}\left(m_{n}, m_{n+2 r}\right) \leq d_{\zeta}\left(m_{0}, m_{1}\right)\left[\zeta\left(m_{n}, m_{n+1}\right) \lambda^{n}\right. \\
& \left.\quad+\zeta\left(m_{n+2}, m_{n+3}\right) \lambda^{n+1}\right]+\sum_{i=1}^{r-2} \prod_{j=1}^{i} \zeta\left(m_{2 j}, m_{n+2 r}\right) \\
& \quad \times \zeta\left(m_{2 i}, m_{2 i+1}\right) \lambda^{2 i} d_{\zeta}\left(m_{0}, m_{1}\right) \\
& \quad+\sum_{i=1}^{r-2} \prod_{j=1}^{i} \zeta\left(m_{2 j+1}, m_{n+2 r}\right) \zeta\left(m_{2 i+1}, m_{2 i+2}\right) \\
& \quad \times \lambda^{2 i+1} d_{\zeta}\left(m_{0}, m_{1}\right)+\prod_{i=1}^{r-1} \zeta \\
& \quad \cdot\left(m_{n+2 j}, m_{n+2 r}\right) \lambda^{n+2 r-2} d_{\zeta}\left(m_{0}, m_{2}\right) .
\end{aligned}
$$

Since $\sup _{q \geq 1} \lim _{i \longrightarrow \infty} \zeta\left(m_{i}, m_{q}\right)\left(\zeta\left(m_{i+1}, m_{i+2}\right) / \zeta\left(m_{i-1}\right.\right.$, $\left.\left.m_{i}\right)\right) \lambda<1$, the series

$$
\begin{gathered}
\sum_{i=1}^{\infty} \prod_{j=1}^{i} \zeta\left(m_{2 j}, m_{n+2 r}\right) \zeta\left(m_{2 i}, m_{2 i+1}\right) \lambda^{2 i}, \\
\sum_{i=1}^{\infty} \prod_{j=1}^{i} \zeta\left(m_{2 j+1}, m_{n+2 r}\right) \zeta\left(m_{2 i+1}, m_{2 i+2}\right) \lambda^{2 i+1},
\end{gathered}
$$

converge by the ratio test. Let

$$
\begin{gathered}
\mathcal{S}=\sum_{i=1}^{\infty} \prod_{j=1}^{i} \zeta\left(m_{2 j}, m_{n+2 r}\right) \zeta\left(m_{2 i}, m_{2 i+1}\right) \lambda^{2 i} \\
\mathcal{S}_{n}=\sum_{i=1}^{n} \prod_{j=1}^{i} \zeta\left(m_{2 j}, m_{n+2 r}\right) \zeta\left(m_{2 i}, m_{2 i+1}\right) \lambda^{2 i} \\
\mathcal{S}^{\prime}=\sum_{i=1}^{\infty} \prod_{j=1}^{i} \zeta\left(m_{2 j+1}, m_{n+2 r}\right) \zeta\left(m_{2 i+1}, m_{2 i+2}\right) \lambda^{2 i+1} \\
\mathcal{S}_{n}^{\prime}=\sum_{i=1}^{n} \prod_{j=1}^{i} \zeta\left(m_{2 j+1}, m_{n+2 r}\right) \zeta\left(m_{2 i+1}, m_{2 i+2}\right) \lambda^{2 i+1}
\end{gathered}
$$

Then, equation (31) takes the following form:

$$
\begin{aligned}
& d_{\zeta}\left(m_{n}, m_{n+2 r}\right) \leq d_{\zeta}\left(m_{0}, m_{1}\right)\left[\zeta\left(m_{n}, m_{n+1}\right) \lambda^{n}\right. \\
& \left.\quad+\zeta\left(m_{n+2}, m_{n+3}\right) \lambda^{n+1}\right]+d_{\zeta}\left(m_{0}, m_{1}\right)\left[\mathcal{S}_{r-1}-\mathcal{S}_{n+1}\right] \\
& \quad+d_{\zeta}\left(m_{0}, m_{1}\right)\left[\delta_{r-1}^{\prime}-\mathcal{S}_{n+1}^{\prime}\right] \\
& \quad+\prod_{i=1}^{r-1} \zeta\left(m_{n+2 j}, m_{n+2 r}\right) \lambda^{n+2 r-2} d_{\zeta}\left(m_{0}, m_{1}\right) .
\end{aligned}
$$

By taking limit $n \longrightarrow \infty$ in equation (35), we get

$$
\lim _{n \longrightarrow \infty} d_{\zeta}\left(m_{n}, m_{n+2 r}\right)=0
$$

Hence, in both cases, $\lim _{n \longrightarrow \infty} d_{\zeta}\left(m_{n}, m_{n+\rho}\right)=0$, 
which shows that $\left\{m_{n}\right\}$ is a Cauchy sequence. As $\mathscr{M}$ is $\mathbf{f}$ -orbitally complete, so there exists $\mu \in \mathscr{M}$ such that $\lim _{n \rightarrow \infty} m_{n}=\mu$. Next, we show that $\mu$ is a fixed point of $\mathbf{f}$. As $\mathbf{f}$ is orbitally continuous, so we have

$$
\begin{aligned}
d_{\zeta}(\mu, \mathbf{f} \mu) \leq & \zeta\left(\mu, m_{n}\right) d_{\zeta}\left(\mu, m_{n}\right)+\zeta\left(m_{n}, m_{n+1}\right) d_{\zeta}\left(m_{n}, m_{n+1}\right) \\
& +\zeta\left(m_{n+1}, \mathbf{f} \mu\right) d_{\zeta}\left(m_{n+1}, \mathbf{f} \mu\right)
\end{aligned}
$$

Since for each $m \in \mathscr{M}, \quad \lim _{n \rightarrow \infty} \zeta\left(m_{n}, m\right)$ and $\lim _{n \longrightarrow \infty} \zeta\left(m, m_{n}\right)$ exist and are finite, so by taking limit $n \longrightarrow \infty$ and using equation (17), we get

$$
\lim _{n \rightarrow \infty} d_{\zeta}(\mu, \mathbf{f} \mu)=0
$$

Therefore, $\mathbf{f} \mu=\mu$. Hence, $\mu$ is a fixed point of $\mathbf{f}$. In view of Lemma $10, m$ is the unique fixed point of $\mathbf{f}$.

Example 4. Let $X=[1,2]$. Define $d_{\zeta}: \mathscr{M} \times \mathscr{M} \longrightarrow[0, \infty)$ by $d_{\zeta}\left(m_{1}, m_{2}\right)=\left(m_{1}-m_{2}\right)^{2}$. Then, $\left(\mathscr{M}, d_{\zeta}\right)$ is a complete controlled rectangular metric space with $\zeta: \mathscr{M} \times \mathscr{M} \longrightarrow[1$, $\infty)$ defined as $\zeta\left(m_{1}, m_{2}\right)=3 m_{1}+2 m_{2}+5$. Define a mapping f : $\mathscr{M} \longrightarrow \mathscr{M}$ by

$$
\mathbf{f} m=\frac{m}{4}
$$

Clearly, all the axioms of Theorem 12 are satisfied, and hence, $m=0$ is a fixed point of $\mathbf{f}$.

Corollary 13. Let $\mathbf{f}: \mathscr{M} \longrightarrow \mathscr{M}$ be a mapping on a complete controlled rectangular metric space $\left(\mathscr{M}, d_{\zeta}\right)$. Suppose that the following axioms hold:

(i) For all $m_{1}, m_{2} \in \mathscr{M}$, we have

$$
d_{\zeta}\left(\mathbf{f} m_{1}, \mathbf{f} m_{2}\right) \leq \lambda d_{\zeta}\left(m_{1}, m_{2}\right), \quad \lambda \in[0,1) .
$$

(ii) $\sup _{q \geq 1} \lim _{i \longrightarrow \infty} \zeta\left(m_{i}, m_{q}\right)\left(\zeta\left(m_{i+1}, m_{i+2}\right) / \zeta\left(m_{i-1}\right.\right.$, $\left.\left.m_{i}\right)\right) \lambda<1$, for any $m_{n} \in \mathscr{M}$

(iii) $\mathbf{f}$ is continuous

Then, $\mathbf{f}$ has a unique fixed point.

Remark 14.

(i) By putting $\zeta\left(m_{1}, m_{2}\right)=s$, for all $m_{1}, m_{2} \in \mathscr{M}$ in Theorem 12, we get Theorem 2.1 of George et al. [22]

(ii) By putting $\zeta\left(m_{1}, m_{2}\right)=1$, for all $m_{1}, m_{2} \in \mathscr{M}$ in Theorem 12, we get the following corollary in view of Das and Dey [27]
Corollary 15. Let $\mathbf{f}: \mathscr{M} \longrightarrow \mathscr{M}$ be a mapping on a rectangular metric space $\left(\mathscr{M}, d_{\zeta}\right)$. Suppose that the following axioms hold:

(i) For all $m_{1}, m_{2} \in \mathscr{M}, d_{\zeta}\left(\mathbf{f} m_{1}, \mathbf{f} m_{2}\right) \leq \lambda d_{\zeta}\left(m_{1}, m_{2}\right)$, where $\lambda \in[0,1)$

(ii) $\left(\mathscr{M}, d_{\zeta}\right)$ is f-orbitally complete

(iii) $\mathbf{f}$ is orbitally continuous

Then, $\mathbf{f}$ has a unique fixed point.

Theorem 16. Let $\mathbf{f}: \mathscr{M} \longrightarrow \mathscr{M}$ be a mapping on complete controlled rectangular metric space $\left(\mathscr{M}, d_{\zeta}\right)$, which satisfies the following axioms:

(i) For all $m_{1}, m_{2} \in \mathscr{M}$, we have

$$
d_{\zeta}\left(\mathbf{f} m_{1}, \mathbf{f} m_{2}\right) \leq \lambda\left[d_{\zeta}\left(m_{1}, \mathbf{f} m_{1}\right)+d_{\zeta}\left(m_{2}, \mathbf{f} m_{2}\right)\right]
$$

where $\lambda \in[0,1 / 2)$.

(ii) $\sup _{q \geq 1} \lim _{i \longrightarrow \infty} \zeta\left(m_{i}, m_{q}\right)\left(\zeta\left(m_{i+1}, m_{i+2}\right) / \zeta\left(m_{i-1}\right.\right.$, $\left.\left.m_{i}\right)\right) \lambda<1$, for any $m_{n} \in \mathscr{M}$, where $\lambda \neq 1 / \zeta\left(m_{1}, m_{2}\right)$ for each $m_{1}, m_{2} \in \mathscr{M}$

(iii) For each $m \in \mathscr{M}, \quad \lim _{n \longrightarrow \infty} \zeta\left(m_{n}, m_{n+1}\right) \leq 1$, $\lim _{n \rightarrow \infty} \zeta\left(m_{n}, \mu\right)$, and $\lim _{n \rightarrow \infty} \zeta\left(\mu, m_{n}\right)$ exist and are finite

Then, $\mathbf{f}$ has a unique fixed point in $\mathscr{M}$.

Proof. Let us take an arbitrary element $m_{0} \in \mathscr{M}$ and choose $m_{1}=\mathbf{f} m_{0}$ and $m_{2}=\mathbf{f} m_{1}$. Then, from equation (41), we obtain

$$
\begin{aligned}
d_{\zeta}\left(m_{1}, m_{2}\right)= & d_{\zeta}\left(\mathbf{f} m_{0}, \mathbf{f} m_{1}\right) \leq \lambda\left[d_{\zeta}\left(m_{0}, \mathbf{f} m_{0}\right)\right. \\
& \left.+d_{\zeta}\left(m_{1}, \mathbf{f} m_{1}\right)\right]=\lambda\left[d_{\zeta}\left(m_{0}, m_{1}\right)\right. \\
& \left.+d_{\zeta}\left(m_{1}, m_{2}\right)\right] .
\end{aligned}
$$

This implies that

$$
d_{\zeta}\left(m_{1}, m_{2}\right) \leq \frac{\lambda}{1-\lambda} d_{\zeta}\left(m_{0}, m_{1}\right)
$$

where $\omega=\lambda /(1-\lambda)<1$, as $\lambda<1 / 2$. By recursively applying equation (41), we obtain

$$
d_{\zeta}\left(m_{n}, m_{n+1}\right) \leq \omega^{n} d_{\zeta}\left(m_{0}, m_{1}\right)
$$

Thus, by taking the limit in equation (44), we have

$$
\lim _{n \rightarrow \infty} d_{\zeta}\left(m_{n}, m_{n+1}\right)=0
$$


Again from equation (41), we have

$$
\begin{aligned}
d_{\zeta}\left(m_{n}, m_{n+2}\right)= & d_{\zeta}\left(\mathbf{f} m_{n-1}, \mathbf{f} m_{1 n+1}\right) \leq \lambda\left[d_{\zeta}\left(m_{n-1}, \mathbf{f} m_{n-1}\right)\right. \\
& \left.+d_{\zeta}\left(m_{n+1}, \mathbf{f} m_{n+1}\right)\right]=\lambda\left[d_{\zeta}\left(m_{n-1}, m_{n}\right)\right. \\
& \left.+d_{\zeta}\left(m_{n+1}, m_{n+2}\right)\right] .
\end{aligned}
$$

By using equation (45), we obtain

$$
\lim _{n \rightarrow \infty} d_{\zeta}\left(m_{n}, m_{n+2}\right)=0
$$

Now, we will show that $\left\{m_{n}\right\}$ is a Cauchy sequence. By following the same procedure as in the proof of Theorem 12 and using equations (45) and (47), we conclude that \{ $\left.m_{n}\right\}$ is a Cauchy sequence. As $\mathscr{M}$ is complete, so there exists $\mu \in \mathscr{M}$ such that

$$
\lim _{n \rightarrow \infty} d_{\zeta}\left(m_{n}, \mu\right)=0
$$

Next, we show that $\mu$ is a fixed point of $\mathbf{f}$. From condition (iii) of Definition 6 , for any $n \in \mathbb{N}$, we have

$$
\begin{aligned}
d_{\zeta}(\mu, \mathbf{f} \mu) \leq & \zeta\left(\mu, m_{n}\right) d_{\zeta}\left(\mu, m_{n}\right)+\zeta\left(m_{n}, m_{n+1}\right) d_{\zeta}\left(m_{n}, m_{n+1}\right) \\
& +\zeta\left(m_{n+1}, \mathbf{f} \mu\right) d_{\zeta}\left(m_{n+1}, \mathbf{f} \mu\right) \leq \zeta\left(\mu, m_{n}\right) d_{\zeta}\left(\mu, m_{n}\right) \\
& +\zeta\left(m_{n}, m_{n+1}\right) d_{\zeta}\left(m_{n}, m_{n+1}\right)+\zeta\left(m_{n+1}, \mathbf{f} \mu\right) d_{\zeta} \\
& +\left(\mathbf{f} m_{n}, \mathbf{f} \mu\right) \leq \zeta\left(\mu, m_{n}\right) d_{\zeta}\left(\mu, m_{n}\right) \\
& +\zeta\left(m_{n}, m_{n+1}\right) d_{\zeta}\left(m_{n}, m_{n+1}\right) \\
& +\zeta\left(m_{n+1}, \mathbf{f} \mu\right) \lambda\left[d_{\zeta}\left(m_{n}, \mathbf{f} m_{n}\right)+d_{\zeta}(\mu, \mathbf{f} \mu)\right]
\end{aligned}
$$

$\lambda \neq 1 / \zeta\left(m_{1}, m_{2}\right)$ for each $m_{1}, m_{2} \in \mathscr{M}$. This implies that

$$
\begin{aligned}
d_{\zeta}(\mu, \mathbf{f} \mu) \leq & \frac{\zeta\left(\mu, m_{n}\right)}{\left(1-\lambda \zeta\left(m_{n+1}, \mathbf{f} \mu\right)\right)} d_{\zeta}\left(\mu, m_{n}\right) \\
& +\frac{\zeta\left(m_{n}, m_{n+1}\right)+\lambda \zeta\left(m_{n+1}, \mathbf{f} \mu\right)}{\left(1-\lambda \zeta\left(m_{n+1}, \mathbf{f} \mu\right)\right)} d_{\zeta}\left(m_{n}, m_{n+1}\right)
\end{aligned}
$$

For each $m \in \mathscr{M}, \lim _{n \longrightarrow \infty} \zeta\left(m_{n}, m_{n+1}\right) \leq 1, \lim _{n \rightarrow \infty} \zeta($ $\left.m_{n}, m\right)$, and $\lim _{n \rightarrow \infty} \zeta\left(m, m_{n}\right)$ exist and are finite. Therefore, by taking limit $n \longrightarrow \infty$ in equation (50) and using equations (47) and (48), we obtain

$$
d_{\zeta}(\mu, \mathbf{f} \mu)=0
$$

which implies that $\mathbf{f} \mu=\mu$. For the uniqueness, let $\nu$ be another fixed point of $\mathbf{f}$ and $\mu \neq v$. From equation (41), we obtain

$$
\begin{aligned}
d_{\zeta}(\mu, v) & =d_{\zeta}(\mathbf{f} \mu, \mathbf{f} v) \leq \lambda\left[d_{\zeta}(\mu, \mathbf{f} \mu)+d_{\zeta}(\nu, \mathbf{f} v)\right] \\
& =\lambda\left[d_{\zeta}(\mu, \mu)+d_{\zeta}(\nu, v)\right],
\end{aligned}
$$

where $d_{\zeta}(\mu, \mu)=0$ and $d_{\zeta}(\nu, \nu)=0$. Hence, from the above inequality, we obtain $d_{\zeta}(\mu, v)=0$, that is, $\mu=\nu$, and $\mu$ is a unique fixed point of $\mathbf{f}$.

\section{Application}

In this section, we will apply Corollary 13 to prove the existence and uniqueness of a solution for the following Fredholm integral equation:

$$
m(t)=\int_{a}^{b} \tau(t, r, m(r)) \mathrm{d} r+v(t), \quad \text { for } t, r \in[a, b]
$$

where $v:[a, b] \longrightarrow \mathbb{R}$ and $\tau:[a, b] \times[a, b] \times \mathbb{R} \longrightarrow \mathbb{R}$ both are continuous functions. Let $\mathscr{M}=\mathbb{C}([a, b])$ be the space of all continuous real-valued functions defined on the closed interval $[a, b]$. Consider

$$
d_{\zeta}\left(m_{1}, m_{2}\right)=\sup _{t \in[a, b]}\left|m_{1}(t)-m_{2}(t)\right|^{2}
$$

Clearly, $\left(\mathscr{M}, d_{\zeta}\right)$ is a complete controlled rectangular metric space with $\zeta: \mathscr{M} \times \mathscr{M} \longrightarrow[1, \infty)$ defined as $\zeta\left(m_{1}\right.$, $\left.m_{2}\right)=3\left|m_{1}(t)\right|+2\left|m_{2}(t)\right|+5$. Next, we will prove our result as follows.

Theorem 17. For all $m_{1}, m_{2} \in \mathscr{M}$ and $t, r \in[a, b]$, the following condition holds:

$$
\left|\tau\left(t, r, m_{1}(r)\right)-\tau\left(t, r, m_{2}(r)\right)\right| \leq \frac{1}{2(b-a)}\left|m_{1}(r)-m_{2}(r)\right|
$$

Then, the integral equation (53) has a unique solution.

Proof. Define f : $\mathscr{M} \longrightarrow \mathscr{M}$ by

$$
\mathbf{f} m(t)=\int_{a}^{b} \tau(t, r, m(r)) \mathrm{d} r+v(t), \quad \text { for } t, r \in[a, b],
$$

where $v:[a, b] \longrightarrow \mathbb{R}$ and $\tau:[a, b] \times[a, b] \times \mathbb{R} \longrightarrow \mathbb{R}$ both are continuous functions. Clearly, $\gamma$ is a fixed point of $\mathbf{f}$, if and only if $\gamma$ is a solution of the integral equation (53). For all $m_{1}, m_{2} \in \mathscr{M}$, we have

$$
\begin{aligned}
& \left|\mathbf{f} m_{1}(t)-\mathbf{f} m_{2}(t)\right|^{2}=\mid \int_{a}^{b}\left[\tau\left(t, r, m_{1}(r)\right)-\left.\tau\left(t, r, m_{2}(r)\right) \mathrm{d} r\right|^{2}\right. \\
& \quad \leq \frac{1}{4(b-a)^{2}} \sup _{s \in[a, b]}\left|m_{1}(s)-m_{2}(s)\right|^{2}\left(\int_{a}^{b} \mathrm{~d} s\right)^{2} \\
& \quad \leq \frac{1}{4} d_{\zeta}\left(m_{1}, m_{2}\right) .
\end{aligned}
$$


It implies that

$$
d_{\zeta}\left(\mathbf{f} m_{1}, \mathbf{f} m_{2}\right) \leq \frac{1}{4} d_{\zeta}\left(m_{1}, m_{2}\right)
$$

where $\lambda=1 / 4 \in(0,1)$. Thus, all the conditions of Corollary 13 are satisfied. Hence, $\mathbf{f}$ has a unique fixed point; that is, the Fredholm integral equation (53) has a solution.

\section{Data Availability}

The data used to support the findings of this study are available from the corresponding author upon request.

\section{Conflicts of Interest}

The authors declare no conflict of interest.

\section{Authors' Contributions}

All authors contributed equally in writing this article. All authors read and approved the final manuscript.

\section{Acknowledgments}

The fourth author would like to acknowledge that his contribution to this work was carried out with the aid of a grant from the Carnegie Corporation provided through the African Institute for Mathematical Sciences.

\section{References}

[1] E. Karapinar, "A short survey on the recent fixed point results on b-metric spaces," Constructive Mathematical Analysis, vol. 1, no. 1, pp. 15-44, 2018.

[2] A. S. Babu, T. Dosenović, M. D. Mustaqali, S. Radenović, and K. P. R. Rao, "Presić type results in bâdislocated metric spaces," Constructive Mathematical Analysis, vol. 2, no. 1, pp. 40-48, 2019.

[3] E. Karapinar, S. Czerwik, and H. Aydi, " $(\alpha, \psi)$-Meir-Keeler contraction mappings in generalized b-metric spaces," Journal of Function spaces, vol. 2018, Article ID 3264620, 4 pages, 2018.

[4] M. Nazam, C. Park, O. Acar, and M. Arshad, "On solution of a system of differential equations via fixed point theorem," Journal of Computational Analysis and Applications, vol. 27, no. 3, pp. 417-426, 2019.

[5] H. Aydi, E. Karapinar, and W. Shatanawi, "Coupled fixed point results for $(\psi, \varphi)$-weakly contractive condition in ordered partial metric spaces," Computers and Mathematics with Applications, vol. 62, no. 12, pp. 4449-4460, 2011.

[6] Z. Kadelburg and S. Radenović, "Notes on some recent papers concerning F-contractions in b-metric spaces," Constructive Mathematical Analysis, vol. 1, no. 2, pp. 108-112, 2018.

[7] V. Parvaneh, M. R. Haddadi, and H. Aydi, "On best proximity point results for some type of mappings," Journal of Function Spaces, vol. 2020, Article ID 6298138, 6 pages, 2020.

[8] S. Banach, "Sur les opérations dans les ensembles abstraits et leur application aux équations intégrales," Fundamenta Mathematicae, vol. 3, pp. 133-181, 1922.
[9] T. Kamran, M. Samreen, and Q. U. Ain, "A generalization of $b$ -metric space and some fixed point theorems," Mathematics, vol. 5, 2017.

[10] S. Czerwik, "Contraction mappings in b-metric spaces," Acta Mathematica et Informatica Universitatis Ostraviensis, vol. 1, pp. 5-11, 1993.

[11] I. A. Bakhtin, "The contraction mapping principle in almost metric spaces," Functional Analysis, vol. 30, pp. 26-37, 1989.

[12] N. Mlaiki, H. Aydi, N. Souayah, and T. Abdeljawad, "Controlled metric type spaces and the related contraction principle," Mathematics, vol. 6, no. 10, p. 194, 2018.

[13] T. Abdeljawad, N. Mlaiki, H. Aydi, and N. Souayah, "Double controlled metric type spaces and some fixed point results," Mathematics, vol. 6, no. 12, p. 320, 2018.

[14] H. R. Marasi and H. Aydi, "Existence and uniqueness results for two-term nonlinear fractional differential equations via a fixed point technique," Journal of Mathematics, vol. 2021, Article ID 6670176, 7 pages, 2021.

[15] W. Shatanawi, A. Mukheimer, and K. Abodayeh, "Some fixed point theorems in extended b-metric spaces," Applied Mathematics and Physics, vol. 80, pp. 71-78, 2018.

[16] Q. Kiran, N. Alamgir, N. Mlaiki, and H. Aydi, "On some new fixed point results in complete extended $b$-metric spaces," Mathematics, vol. 7, no. 5, p. 476, 2019.

[17] N. Alamgir, Q. Kiran, H. Isik, and H. Aydi, "Fixed point results via a Hausdorff controlled type metric," Advances in Difference Equations, vol. 2020, no. 1, Article ID 24, 2020.

[18] L. Subashi and N. Gjini, "Some results on extended $b$-metric spaces and Pompeiu-Hausdorff metric," Journal of Progressive Research in Mathematics, vol. 12, pp. 2021-2029, 2017.

[19] B. Alqahtani, A. Fulga, and E. Karapinar, "Non-unique fixed point results in extended b-metric space," Mathematics, vol. 6, pp. 1-11, 2018.

[20] A. Branciari, "A fixed point theorem of Banach-Caccioppoli type on a class of generalized metric spaces," Publicationes Mathematiques, vol. 57, pp. 31-37, 2000.

[21] M. Asim, M. Imdad, and S. Radenović, "Fixed point results in extended rectangular $b$-metric spaces with an application," UPB Scientific Bulletin Series A., vol. 20, pp. 43-50, 2019.

[22] R. George, S. Radenović, K. P. Reshma, and S. Shukla, "Rectangular b-metric space and contraction principles," Journal of Nonlinear Sciences and Applications, vol. 8, no. 6, pp. 10051013, 2015.

[23] N. Mlaiki, M. Hajji, and T. Abdeljawad, "A new extension of the rectangular -metric spaces," Advances in Mathematical Physics, vol. 2020, Article ID 8319584, 7 pages, 2020.

[24] I. R. Sarma, J. M. Rao, and S. S. Rao, "Contractions over generalized metric spaces," Journal of Nonlinear Sciences and Applications, vol. 2, no. 3, pp. 180-182, 2009.

[25] B. Samet, "Discussion on "A fixed point theorem of BanachCaccioppoli type on a class of generalized metric spaces" by A Branciari," Publicationes Mathematicae Debrecen, vol. 76, pp. 493-494, 2010.

[26] T. Suzuki, "Generalized metric spaces do not have the compatible topology," Abstract and Applied Analysis, vol. 2014, Article ID 458098, 5 pages, 2014.

[27] P. Das and L. K. Dey, "A fixed point theorem in a generalized metric space," Soochow Journal of Mathematics, vol. 33, pp. 33-39, 2007. 